\title{
Quality of Antenatal Care Services in South-Western Uganda
}

\author{
Kennedy Sakaya Barasa ${ }^{1, *}$, Jones Mutune Mutua ${ }^{2}$ \\ ${ }^{1}$ Deparment of Statistics and Actuarial Sciences, Jomo Kenyatta University of Science and Technology, Nairobi, Kenya \\ ${ }^{2}$ Department of Mathematics and Statistics, University of Missouri - Kansas City, Kansas City, USA
}

Email address:

sakayak33@gmail.com (K. S. Barasa),mutuajo87@gmail.com (J. M. Mutua)

\section{To cite this article:}

Kennedy Sakaya Barasa, Jones Mutune Mutua. Quality of Antenatal Care Services in South-Western Uganda. American Journal of Health Research. Vol. 3, No. 5, 2015, pp. 279-285. doi: 10.11648/j.ajhr.20150305.13

\begin{abstract}
Objectives: The study aimed at the evaluation of the quality of antenatal care services in South Western Uganda Methods: This research used a data set from the Population Council of Kenya, which was conducted in South-Western Uganda, and it drew a representative sample of health facilities, by administering a questionnaire and making an assessment of the facilities. The sample size was selected using a stratified; two stage cluster design and enumeration areas were the sampling units for the first stage. 31 facilities were included in the study drawn from 9 districts and among the facilities that were assessed, 5 were government owned, 17 were privately run and 9 were faith-based facilities. 15 facilities were clinic, 10 were health centers, and 5 were hospitals and only 1 dispensary. Data Analysis: The data was analyzed using IBM SPSS version 20. Findings: This project found out that, the intervention facilities offered more ANC services than control. Majority of facilities lacked essentials ANC drugs - only $2.0 \%$ of intervention facilities had delivery equipment. Except for cancer screening and child delivery supplies, facilities had essential supplies and equipment and recommended infrastructure (including a private ANC room for consultation) for provision of ANC services. Significantly different, facilities under control group were well supplied with ARV drugs. For service charges, control group charged more of the two except for caesarian delivery method which was not covered by the voucher program. Conclusion: Quality of care was high. The intervention program increased client satisfaction with ANC services received though little evidence exists on the effect the program on quality of ANC services. Low Socio-Economic status limits access to ANC services. Vouchers were consumed more by clients of low socio-economic status and there were no much difference between the two sets of facilities is evident.
\end{abstract}

Keywords: Antenatal Care, Intervention Facilities, Control Facilities, Quality of Care, Uganda

\section{Introduction}

It is estimated that every year 3 million newborn babies die within the first month of life and between 2.1 to 3.8 million babies are stillborn (UNICF, 2012) Antenatal care (ANC) is one of the recommended interventions to help reduce these alarming maternal and newborn mortalities(Yakoob et al, 2010). Most low-income countries have adopted a focused ANC strategy promoted by WHO which includes four visits and structured elements within these.

Quality of care was conceptualized at the International Conference on Population and Development in 1994 as a means to assist productive couples in achieving their fertility intentions through access to improved family planning programs. Recently, it has grown and more concepts of quality of care have been largely applied to include other sexual and reproductive health services including antenatal care (WHO, 2011). This, among other efforts, aims at delivering safe motherhood which is critical for positive pregnancy results. Antenatal care, ANC, a component of SM, focuses on birth preparedness and readiness to handle complications.

The primary aim of ANC is ensuring prevention, early detection and prompt management of pregnancy related complications. This period presents opportunities for reaching pregnant women with a number of interventions that may be vital to their health and well-being and that of their infants.

Much has been done comparing QOC between public sector and private facilities worldwide but studies related to voucher programs intervention are limited. Studies have focused on family planning component of SM leaving behind ANC component among others. Although these studies provide significant contributions, most of them lack a comprehensive approach of including lay person's perception QOC. 
Assessment of QOC is on the basis of the framework of quality of care. We evaluate QOC in facilities implementing an intervention program (Voucher and Accreditation program) and those not considered in the program and compare their outcome and patient's perception towards the services provided. In order to complement and improve the framework, it is proposed that the lay persons' (and providers') perspective should be included in the definition of quality of care as a metric to the level of QOC expected by them. This could explain why developing countries report high levels of quality care but still have the highest mortality rate in the world. Uganda, being a country of SSA, mortality rate remains high (UNECA/AfDB, 2010) and thus making it an appropriate case study from Low and Middle Income Countries (LMIC).

This study mainly assesses the quality of available and accessible antenatal health care in South Western Uganda. The study also aims at evaluating the quality of antenatal care services, assessing the effect of the voucher and accreditation interventions on access to quality of ANC services, evaluating the relationship between QOC received and client satisfaction with quality of ANC Services provided, and determining the relationship between socio-economic status and voucher utilization.

\subsection{Statement of the Problem}

Most of world's maternal deaths occur in developing world though majority of deaths and are avoidable. Over the years, efforts to reduce maternal mortality and morbidity have included promoting antenatal care, providing family planning services, and improving essential obstetric care. Despite such interventions, maternal mortality rates remain high for Uganda. There is need to assess whether the interventions made are efficient enough to reduce the maternal mortality rates through improved quality of care in health facilities.

\subsection{Objectives}

\subsubsection{General Objective}

This study reports evaluation of the quality of antenatal care services in South Western Uganda.

\subsubsection{Specific Objectives}

1. To evaluate the quality measures of antenatal care services and assess the effect of the voucher and accreditation interventions on access to and quality of ANC services South Western Uganda.

2. To evaluate the relationship between QOC received and client satisfaction with quality of ANC Services provided.

3. To determine the relationship between socio-economic status and voucher utilization.

\subsection{Limitations of the Study}

This study had a few limitations. The study mainly focused on Uganda, a developing country. The results were unique to low and middle income countries and thus the findings cannot be replicated in high income countries.

\section{Reviews of Previous Studies}

The London School of Hygiene and Tropical Medicine conducted several studies to evaluate quality of health care in Tanzania, Papua New Guinea, and Brazil. Almost all of the recent studies of quality of care in health care seem to broadly follow the structural and process approaches to quality evaluation. In Dar es Salaam, Tanzania, it was assessed that three different types of quality in primary curative care; structural, technical, and interpersonal relations could be used. The study concluded that compared to government clinics, the study confirmed that user satisfaction was higher at voluntary agency clinics where clients perceived better clinical performance and interpersonal relations with providers (Kanji, et al., 1992).

In Papua New Guinea, (Garner, et al., 1990) evaluates the quality of health facilities in rural Papua New Guinea by examining structure and process dimensions of quality of care. The results indicate that there were no formally established standards of care before the assessment commenced and thus expected care levels were determined using local experts with extensive experience in rural health services in the country. Service components that were used as part of the quality of care assessment were physical facilities, staff performance, level of supervision and availability of equipment and drugs. The findings from the standard management protocols of inpatients reflected how patient notes were poorly recorded, disease severity and outcomes were not recorded.

In comparing the quality of ANC between public and private providers in Tanzania, (Boller, et al., 2003) noted that both sets of facilities had good structural and interpersonal aspects of Quality of Care. However, both had poor technical aspects. In general, private providers were better than public providers in aggregated aspects.

Irrespective of whether private or public, many ambulatory health care services are delivered poorly but private providers are more client-centered than the public providers in low and middle income countries. Provider competence and patient satisfaction does not vary with provider category, (Berendes, et al., 2011).

A Systematic Review, "The Impact of Vouchers on the Use and Quality of Health Goods and Services in Developing Countries", concludes that health voucher programs have been successful in increasing utilization of health goods/services, targeting specific populations, and improving the quality of services. While these results are encouraging, a subsequent link that voucher programs improve the health of the population was not evident in the data analyzed in that review (Meyer, et al., 2011).

About 99\% of world's maternal deaths occur in developing world though majority of deaths and are avoidable. Over the years, efforts to reduce maternal mortality and morbidity have included providing family planning services, promoting antenatal care, improving essential obstetric care, and addressing the SES of women. Despite such interventions, maternal mortality rates remain 
high for SSA. There is need to assess whether the interventions made are efficient enough to reduce the maternal mortality rates through improved quality of care.

Antenatal care coverage across Africa, however, has seen steady improvement. In 2010 MDG report, 40 African countries recorded a rate higher than the WHO regional average of $73 \%, 18$ of them having 90 percent or more and only 10 countries fell below this average (UNECA/AfDB, 2010). Despite interventions, we have seen maternal mortality still remains high even in SSA countries where utilization of maternal health care services (such as antenatal care) has improved (UNEC/AfDB, 2010). This suggests accessibility without quality improvement is never enough to reduce maternal death.

Millennium Development Goal 5, aiming at improving maternal health, target $5 \mathrm{a}$ aims at reducing the mortality rate by three-quarters, between 1990 and 2015 while target 5 b aims at achieving a universal access to reproductive health. To achieve this goal, it is estimated that an annual decline in maternal mortality of $5.5 \%$ is needed. However, between 1990 and 2005 the annual decline was only $0.5 \%$ in the sub-Saharan region (Ronsmans and Graham, 2006). Meaningful health-based interventions are necessary to accomplish this goal. Regular assessments and progressive reports have to be made to show the advancement made.

\section{Methods}

\subsection{Study Design and Data Source}

This study used data set from the Population Council of Kenya. The Population council conducted a study in South-Western Uganda, and this survey drew a representative sample of health facilities, by administering a questionnaire and making an assessment of the facilities.

This study was a descriptive cross-sectional study design that employed both quantitative parameters and generally cross sectional study that allowed information about the target population to be obtained at that point in time, This design was suitable because it explored all the necessary information regarding the study objectives and covered a good number of the target population that allowed generalization of the information.

The sample size was selected using a stratified; two stage cluster design and enumeration areas were the sampling units for the first stage. Among the facilities assessed, 5 were government owned, 17 were privately run and 9 were faith-based facilities. 15 facilities were clinic, 10 were health centers, and 5 were hospitals and only 1 dispensary.

The study population comprised of pregnant women and mothers between $0-6$ months after delivery at post-natal clinics seeking services for their infants at selected health facilities in South-West Uganda.

\subsection{Statistical Tests}

\subsubsection{Paired $t$ - Test}

T-test is a method based on t-distribution and assumes data collected is from a population that is normally distributed. It is appropriate while judging significance of sample mean or judging significance of difference between two samples mean. In case the samples are related, we use paired t-test test. The test statistic is calculated from the sample data and then compared with its probable value based on t-distribution at a specified level of significance for concerning degrees of freedom of rejecting or not rejecting the null hypothesis.

Once the test statistic is obtained, it is compared with the probability of obtaining the same score due to chance from the t-distribution table. Usually the null hypothesis states that there is no difference between means, whereas the alternative hypothesis states there is a difference. Null hypothesis and alternative hypothesis are represented as $H_{0}: \mu_{1}=\mu_{2}$ and $H_{0}: \mu_{1} \neq \mu_{2}$, respectively, where $\mu_{1}$ is the mean from intervention group, and $\mu_{2}$ is the mean from the control group.

The decision made is rejecting the null hypothesis whenever p-value is less than or equal to 0.05 and conclude that there is no enough evidence to show difference in sample means. Else, we do not reject the null hypothesis implying there was sufficient evidence that there was a difference in sample means. Two tail paired t-test was used to compare variables between intervention group and control group and their p-values were tabulated as well.

Facilities' and providers' preparedness to offer competent high quality of ANC were assessed. The facility inventory assesses the existing infrastructure to provide comprehensive antenatal care services to the population they serve. We compare the intervention and control facilities at baseline to examine whether there are any particular differences in the structural features of quality of care which may influence the delivery of ANC services. To do these, the minimum structural features are compared against the national guidelines as stipulated in the norms and standards document published by the Ministry of Health. Specific requirements for ANC are compared against the WHO ANC model. We employ descriptive statistics and paired t-test to evaluate frequencies, mean scores and to test existence of any statistical differences between the two sets of facilities.

\subsubsection{Regression Model}

Regression analysis as a tool for investigating relationships between variables helps ascertain the causal effect of one variable (called independent) over another (known as dependent) variable. Also, it enables one to assess level of statistical significance of the estimated relationships. Regression modeling constructs equations that can be used to predict results of dependent (outcome) variable for any value of independent (explanatory) variable which have not been tested or observed. It assumes that each of the variables is normally distributed with equal variances. Regression analysis draws a line of best fit through data points of the scatter plot using the least square method.

While comparing client satisfaction with services received, and amount paid for services, we shall get models of the form; 


$$
\begin{gathered}
\text { Service }_{\text {satisfaction }}=\beta_{0}+\beta_{1} \text { Service }_{\text {received }}+e \\
\text { Service }_{\text {satisfaction }}=\beta_{0}+\beta_{1} \text { amount }_{\text {paid }}+e
\end{gathered}
$$

\subsection{Data Analysis}

The data was analyzed using IBM SPSS version 20 and the report was presented in form of tables and graphs.

\section{Results}

This section assesses client socio-demographic characteristics, feeling and satisfaction with service received and payments made. A total of 264 ANC clients were interviewed.

Table 1. Facilities Assessed.

\begin{tabular}{llll}
\hline District & Intervention (\%) & Control (\%) & Total (\%) \\
\hline Bushenyi & 60.0 & 40.0 & 32.3 \\
Ibarara & 57.1 & 42.9 & 22.6 \\
Isingiro & 66.7 & 33.3 & 9.7 \\
Kabale & 100.0 & 0.0 & 3.2 \\
Kirahura & 66.7 & 33.3 & 9.7 \\
Lyantonde & 100.0 & 0.0 & 3.2 \\
Mbarara & 66.7 & 33.3 & 9.7 \\
Ntungamo & 50.0 & 50.0 & 6.5 \\
Sembabule & 100.0 & 0.0 & 3.2 \\
Total & 64.5 & 35.5 & 31 \\
\hline
\end{tabular}

\subsection{Socio-Demographic Characteristics}

Majority of clients were less than thirty years old $(92.02 \%)$ with a median age of 25 and $\operatorname{IQR}(21,30)$. Eighty four percent had education up to primary level, with $3.79 \%$ having tertiary education. Uganda being a Christian majority nation, $97.35 \%$ clients were religious with $92.05 \%$ being Christians. Client's marital status was assessed and the study found out that $96.6 \%$ were married and living together with their partners and for those who were not married, $75 \%$ were in a relationship. This shows majority of pregnancies were as a result of relationships rather than unwanted.

To evaluate exposure to risky sexual practices, questions about co-wives and if partner lived with client were asked and it showed that $8.68 \%$ of clients had partners living elsewhere and $8.97 \%$ had co-wives - even up to five. Majority of poorer clients went to intervention facilities $(60.0 \%)$ compared to those from the poor group (55.25\%). Generally, $57.20 \%$ of clients went to intervention facilities and $84.17 \%$ found it difficult to raise UGS25, 000 if they become ill.

Median (IQR) for monthly household income was UGSh.80000 (50000, 160000) with an average household spending of UGSh.30000 $(20000,60000)$ on all household goods and services and UGSh.10000 $(9500,20000)$ on health services. Clients were asked if they, or their partners, were covered by health insurance and $2.15 \%$ were covered with strong likeliness between those who were visiting intervention facilities and those who were visiting control facilities $(\mathrm{p}=0.925)$.

To evaluate whether the current pregnancy was intended, $77.1 \%$ of clients with pregnancies at that particular time, said they were willing to carry it while $11.24 \%$ said they wanted to wait longer and $11.63 \%$ never wanted it at all. This shows a significant worrying number of unwanted pregnancies. Possibly related to the youthful age of clients, $62.74 \%$ were willing to have another pregnancy in the future.

\subsection{Current Pregnancy}

Concurring with earlier observation, $94.66 \%$ clients had a pregnancy of more than three months and $57.2 \%$ had attended their first ANC service at recommended gestation period (16 weeks). Generally, all clients were served by trained personnel while receiving ANC services. 99.62\% were served by nurse/midwife and $0.78 \%$ by a doctor with no differences between the two groups ( $p$-value $>0.209$ ).

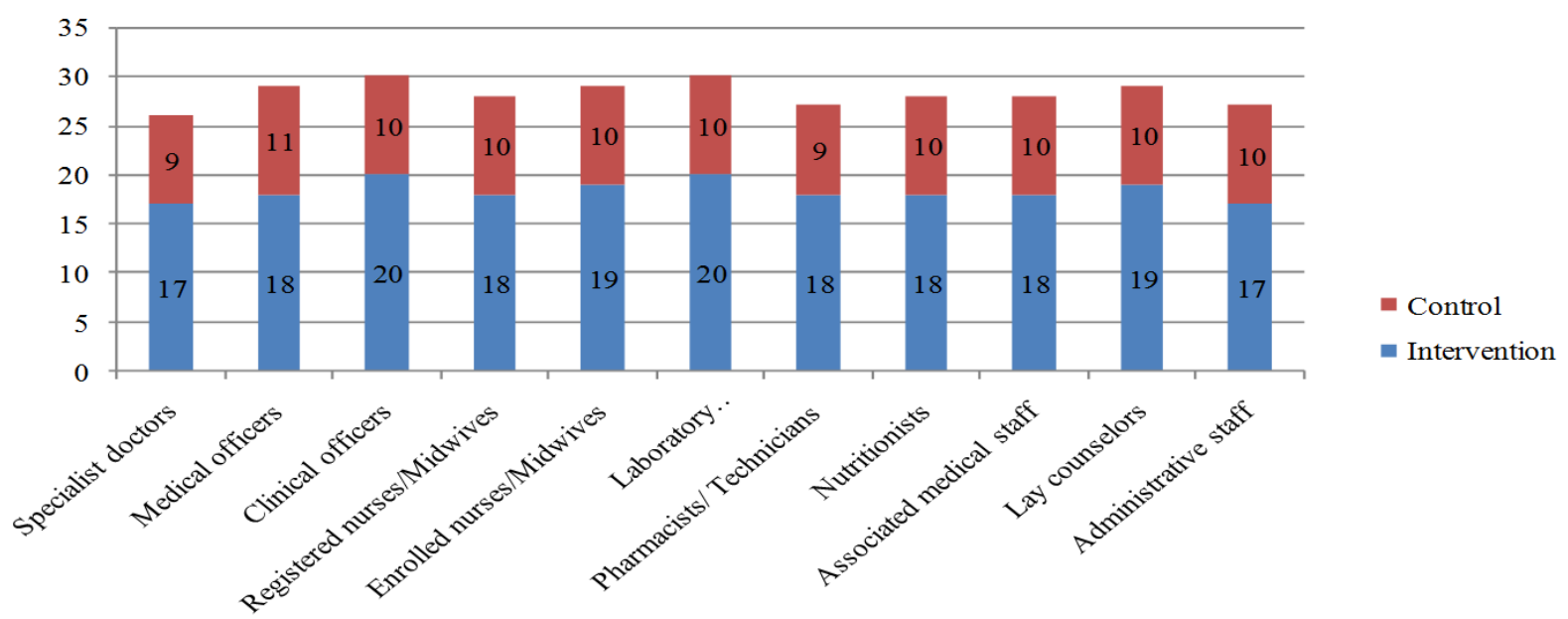

Figure 1. Staffing Levels.

\subsection{Transport to Health Facility}

To assess proximity of health facilities to clients, mode and cost of transport was asked and the study showed that $87.75 \%$ accessed health facility by walking, use of bicycles or motorcycles while the rest used vehicles. Both sets of groups paid similar amounts for transport with $68.42 \%$ paying 
UGS2000 or less. $85.96 \%$ spent two hours or less to reach to the facility. For $33.33 \%$ of clients, distance from residence to health facility was a hindrance to service use while insufficiency of providers at facility was quoted by $8.33 \%$ of clients.

\subsection{Service Received}

On average, $62.59 \%$ of clients from intervention group were attended to by more than one service provider compared to $31.82 \%$ from control group ( $p$-value $<0.001$ ). This supports the claim that there was an overload of nursing staff since $99.62 \%$ of them checked the patients. For tests actually performed on clients, disparity existed. Intervention delivered better services in taking client weight ( $p$-value $=0.001$ ), checking for anemia ( $\mathrm{p}$-value $=0.037$ ), and checking urine for protein and sugar ( $\mathrm{p}$-value $<0.0001)$.

Measuring client weight was also very essential test for FANC. $97.97 \%$ of intervention clients received this service where the control group had $72.97 \%$ of clients with a $\mathrm{p}$-value $<0.001$. Intervention clients also received services like taking blood sample ( $\mathrm{p}$-value $=0.030)$, taking urine sample ( $\mathrm{p}$-value $<0.001$ ), and giving information about STI/AIDS ( $p$-value $=0.012)$ while control clients received more PMTCT information than intervention clients ( $p$-value $=0.047$ ) and information about child spacing and family planning ( $p$-value $=0.025$ ). Out of the 22 considered services, controlled group had a mean score of 11.46 while intervention had 11.41 ( $p$-value $=0.9277)$. More clients from intervention group received more of the services than their control group counterparts $(\mathrm{p}$-value $=0.025)$.

\subsection{Client Satisfaction}

Client satisfaction towards service was evaluated between the two groups. In both cases, majority felt the time spent before being attended to by a service provider was reasonable $(47.49 \%$ and $59.73 \%$ for control and intervention, respectively). Similar results were observed for time spent with service providers $(71.68 \%$ for control and $83.22 \%$ for intervention with a p-value $=0.010) .7 .09 \%$ of all clients paid for the services they received with a $\mathrm{p}$-value and median (IQR) of 0.057 and UGS7000 (3000, 10000), respectively, between the two groups. Service charges were greatly reduced and $55.55 \%$ of clients felt that the charges were affordable. Intervention was $64.29 \%$ of clients compared to $50.00 \%$ of control. However, there was no significant difference between the two groups ( $p$-value $=0.615)$. Clients' satisfaction with services was not different in both groups with control having $40.83 \%$ while intervention projected $59.15 \%$ ( $p$-value $=0.083$ ). Satisfaction reasons included cheap services and of high quality, active and well prepared staff, and free HIV test.

Overall, $82.58 \%$ of clients were satisfied with services received and all $(100.00 \%)$ clients from intervention facilities would recommend the facility to a friend compared to $95.54 \%$ for control group. Clients from intervention group felt more satisfied with facility than clients from control group ( $p$-value $=0.010)$. A regression test proved a positive impact of intervention program on client's satisfaction since we obtained the following values, Pseudo $R^{2}=0.0081$, odds ratio $=1.5812$ and standard error $=0.5151$.

\subsection{Voucher Use}

Disparities exist between the two groups for voucher knowledge and use. There was high knowledge about vouchers with $85.16 \%$ of all clients having had obtained a voucher ( $p$-value $<0.001$ ). $96.00 \%$ bought the vouchers from a distributor. Although a greater percentage (90.84\% total, $99.16 \%$ intervention and $8.33 \%$ control) had used the vouchers obtained, clients highlighted facilities being quite a distance, lack of knowledge on the accredited facilities, and lack of transport as major reasons why they had not used the vouchers. The clients would recommend use of vouchers for health care services to a friend. On cross-tabulation of clients who used voucher against their SES, it was clear those who used voucher were majorly the poorer $(97.96 \%)$ and for those who did not use the voucher $(91.67 \%)$ were poor ( $p$-value $=0.029$ ). This is one indication that showed poverty affected the use of vouchers.

\section{Discussion}

From the study it clearly shows that both sets of facilities were well prepared when it came to delivering ANC services. There were low levels of staffing and there were no exclusive stationing of staff in departments offering maternal and child health care and the staff had received little training in previous twelve months. Providers reported a very high level of knowledge on ANC services despite lack of training and IEC materials. Largely, facilities' staff had not received training for ANC services with intervention being more affected.

HIV/STI integration was exercised in majority of intervention facilities than in control facilities. Other services that were offered by many of facilities included urine tests, sputum test, high blood pressure, malaria, stool and pregnancy tests and the study showed that there was a difference in malaria and stool tests between the two groups with minimal performance by control group.

Intervention facilities offered more ANC services than control facilities and also majority of facilities lacked essentials ANC drugs. Except for cancer screening and child delivery supplies, facilities had essential supplies and equipment and recommended infrastructure (including a private ANC room for consultation) for provision of ANC services. Significantly different, facilities under control group were well supplied with ARV drugs. For service charges, control group charged more of the two except for caesarian delivery method which was not covered by the voucher program.

Possibly related to poor training, client examinations and referrals were poor and TB discussion was poor with control being more affected. PMTCT and STI too were not comprehensively discussed though the providers from intervention group did better. For birth preparedness, discussion on birth planning, danger signs during pregnancy, advice to use skilled health worker during delivery and 
postpartum care were not well talked about. This poses a risk for mothers and newborns when they are most weak. Postpartum care was not recommended by most of providers.

Generally, there was good rapport between the clients and providers and was significantly better in intervention group. There was difference in rapport creation and discussion of ANC services between providers in intervention and those from control group. Clients did not ask questions with intervention group being more affected. However, majority understood information shared.

There were alarming levels of unwanted pregnancies (11.63\%) possibly because most of the clients were married but were not living together with their spouses. ANC visits compliance was higher in intervention group than in control group, and above average number of clients had their first ANC visit within a gestation period of the current pregnancy in both groups. Most clients lived within proximity of health facilities with a great percentage accessing health facilities by motorcycle, bicycle or by foot. Staff overload was evident. Clients were attended to by nurses with more than average being seen by more than one staff in one visit. Clients in intervention facilities received more service, hence higher quality, compared to those who visited control facilities. Clients were satisfied more in intervention than in control facilities with time spent while waiting for service provider, time spent with provider, and ANC services received.

Clients and providers knew about vouchers and a good percentage of clients informed their friends about the use of voucher. On close check, poverty affects the use of voucher. Distance to facilities, not knowing accredited facilities, and lack of transport was major challenges to access of ANC services. Clearly, intervention group outdoes control in performance index from facilities' preparedness, supplies and equipment, provider competence, service delivery to client satisfaction.

Findings of this study highlighted areas of antenatal care services that showed no difference in quality measure between facilities on intervention programs against those not included in programs (control). This helped in emphasizing on stronger goal-specific policies and strategies to improve the intervention programs rather than on items whose objective was already accomplished or would never improve any more.

\section{Conclusions}

Though performance index may favor intervention program, generally there was no difference between quality of ANC services offered in facilities which were enrolled for intervention package and those that were not enrolled for the intervention package (control). The difference in rapport creation could be accrued to presence of research staff and not as a result of intervention program since most of the clients did not actively participate in discussion during the session. Intervention program considerably increases access to ANC services but still more has to be done to improve transport to health facilities. The intervention program increased client satisfaction with ANC services being received and the study also showed that low socio-economic status limits access to
ANC services and that's why vouchers were consumed more by clients with low socio-economic status.

\section{Recommendations}

1. Further studies should be carried out on the same data set using other statistical methods, like probit analysis, to assess the immediate impact of intervention program on outcome variables.

2. There is need to research on high level of unwanted pregnancies considering majority of clients were married or in relationships.

3. Staff training has positive impact on staff knowledge and ANC service delivery discussions therefore the health facilities' staff should be trained frequently on emergent health care issues and on ANC package.

4. Lack of IEC materials negatively impact the discussion session between the service providers and clients therefore to increase client's participation in discussion, they should be well informed prior to service deliver.

\section{Acknowledgements}

We appreciate and thank the Population Council of Kenya for providing us with the necessary materials and data.

\section{Nomenclature}
AfDB - Africa Development Bank
AIDS- Acquired Immune-Deficiency Syndrome
ANC - Antenatal Care
FANC -Focused Antenatal Care
HBP - High Blood Pressure
HIV - Human Immuno-Virus
IEC - Information, Education and Communication
ITBN - Insecticide Treated Bed Nets
MOH- Ministry of Health
PMTCT- Prevention of Mother-to-Child Transmission
PNC- Post-Natal Care1
QOC -Quality of Care
SM - Safe Motherhood
SSA -Sub-Saharan Africa
STI -Sexually transmitted Infections
TB - Tuberculosis
UNECA - United Nations Economic Commission for Africa
VCT - Voluntary Counseling and Testing
VDRL- Venereal Disease Research Laboratory

\section{References}

[1] Berendes, S., Heywood, P., Oliver, S. \& Garner, P., 2011. Quality of Private and Public Ambulatory Health Care in Low and Middle Income Countries: Systematic Review of Comparative Studies. PLoS Med, 8(4)J. Clerk Maxwell, A Treatise on Electricity and Magnetism, 3rd ed., vol. 2. Oxford: Clarendon, 1892, pp.68-73. 
[2] Birungi, H. \& Onyango-Ouma, W., 2006. Acceptability and Sustainability of the WHO Focused Antenatal Care package in Kenya.

[3] Blake, O. \& Wakeling, J., 2011. Muscle Coordination during an Outdoor Cycling Time Trial.

[4] Boller, C., Wyss, K., Mtasiwa, D. \& Tanner, M., 2003. Quality and comparison of antenatal care in public and private providers in the United Republic of Tanzania. Bulletin of the World Health Organization, 81(2).

[5] California Maternal Quality Care Collaborative. Cesarean Deliveries, Outcomes, and Opportunities for Change in California: Toward a Public Agenda for Maternity Care Safety and Quality. Accessed Jul 29, 2015. http://www.cmqcc.org/resources/2079/download.

[6] Campbell, S., Roland, M. \& Buetow, S., 2000. Defining quality of care. Social Science \& Medicine, 51(2000), pp. 1611-1625.

[7] Curhan, R., 1972. The Relationship beween shelf space and unit sales in a supermarket. Journal of Marketing Research, Volume 9, pp. 406-412.

[8] Dehnavi, A. R. M. et al., 2011. Detection and classification of cardiac ischemia using vectorcardiogram signal via neural network.. Journal of Research in Medical Sciences, 16(2), pp. 136-142.

[9] Family Care International, 2007. The Safe Motherhood Initiative Review, New York: s.n.

[10] Hutchinson, P., Do, M. \& Agha, S., 2009. Client Satisfaction And The Quality Of Family Planning Services: A Comparative Analysis Of Public And Private Health Facilities In Ghana, Kenya, And Tanzania, Bethesda: Abt Associates Inc.
[11] Kanji, N., Kilima, P. M. \& Munishi, G. M., 1992. Quality of Primary Curative Care in Dar es Salaam, s.l.: s.n.

[12] Koblinsky, M., Campbell, O. \& Heichelheim, J., n.d. Organizing delivery care: what works for safe motherhood?, s.l.: s.n.

[13] Lucini, D. et al., 2011. Health Promotion in the Workplace: Assessing Stress and Lifestyle With an Intranet Tool. Journal of Medical Internet Research, 13(4).

[14] Mainz, J., 2003. Defining and Classifying Clinical Indicators for Quality Improvement. International Journalfor Quality in Health Care, 15(6), pp. 523-530.

[15] Meyer, C., Bellows, N., Campbell, M. \& Potts, P. M., 2011. The Impact of Vouchers on the Use and Quality of Health Goods and Services in Developing Countries: A Systematic Review.

[16] MOH, 2006. Essential Obstetric Care Manual. s.l.s.s.n.

[17] MOH, 2009. NATIONAL HEALTH POLICY: Reducing poverty through promoting people's health. Kampala: $\mathrm{MOH}$.

[18] Needleman, J., Buerhaus, P., Mattke, S. \& Stewart, M., 2002. Nurse-Staffing Levels And The Quality Of Care In Hospitals. The New England Journal of Medicine, 346(22), pp. 1715-1721.

[19] UNICEF, WHO, The World Bank, United Nations Population Division: Levels and Trends in Child Mortality. New York; 2012.

[20] Yakoob MY, Lawn JE, Darmstadt GL, Bhutta ZA: Stillbirths: epidemiology, evidence, and priorities for action. Semin Perinatol 2010, 34(6):387-394. 